\title{
Diversidad de Vanilla spp. (Orchidaceae) y sus perfiles bioclimáticos en México
}

\author{
Álvaro Flores Jiménez ${ }^{1}$, Delfino Reyes López ${ }^{2 *}$, Daniel Jiménez García ${ }^{1}$, Omar Romero Arenas ${ }^{1}$, \\ José Antonio Rivera Tapia ${ }^{1}$, Manuel Huerta Lara ${ }^{1} \&$ Araceli Pérez Silva ${ }^{3}$ \\ 1. Benemérita Universidad Autónoma de Puebla, Instituto de Ciencias, Centro de Agroecología y Ambiente, Boulevard \\ 14 sur 6301, Ciudad Universitaria, 72570 Puebla, Puebla, México; alvaro_flores-@hotmail.com, \\ daniel.jimenez@correo.buap.mx, biol.ora@hotmail.com,Jart70@yahoo.com, batprofessor@hotmail.com \\ 2. Benemérita Universidad Autónoma de Puebla, Facultad de Ingeniería Agrohidráulica, Av. Universidad s/n San Juan \\ Acateno, Teziutlán, Puebla, México; delfino_reyes2001@yahoo.com.mx, reymidas46@hotmail.com \\ 3. Instituto Tecnológico de Tuxtepec, Av. Dr. Victor Bravo Ahuja s/n, Col. 5 de Mayo, 68350, Tuxtepec, Oaxaca, México; \\ apsilva30@hotmail.com \\ * Correspondence
}

Recibido 02-XI-2016. Corregido 07-IV-2017. Aceptado 08-V-2017.

\begin{abstract}
Diversity and bioclimatic profiles of Vanilla spp. (Orchidaceae) in Mexico. The genus Vanilla comprises around 110 species distributed throughout Earth's tropical regions, with the largest number of reported species growing in the American continent. Vanilla farming is associated with many Mexican cultures such as the Totonac, Mayan, Chinantec, and Mazatec, among others. Currently, this crop is threatened by technical, social, ecological, and climatic conditioning factors, limiting its production and the preservation of wild and cultivated species. It is therefore necessary to ascertain the current diversity status of each of these species, as well as some of their main bioclimatic profile indicators, in order to help decision-making, aimed at preserving and genetically improve these species. During 2008, we gathered data from IPN, MEXU, and XAL herbaria, as well as from CONABIO's World Information Network on Biodiversity (REMIB), the Global Biodiversity and Information Facility (GBIF), and we also used data from live access to BUAP's vanilla germplasm bank, obtained between 2008 and 2014. Distribution maps were generated using a geographical information system. Bioclimatic profiles for each species were obtained considering 19 WorldClim variables and altitude at a spatial resolution of approximately $1 \mathrm{Km}^{2}$. Variance, Mean, and standard deviation for each of the 19 variables were calculated at each of the registered points. Extreme environmental condition intervals (minimum, average, and maximum) were also obtained. In order to determine the most important distribution variables of the species, we performed a principal component analysis and carried out Kruskal-Wallis and Dunn's tests on the variables identified as significant. Results indicated records for $V$. planifolia, V. pompona, V. insignis, V. inodora V. odorata, $V$. cribbiana, and $V$. sprucei in Mexico, distributed throughout nine states in the country. V. planifolia presented wide intervals of temperature and rain precipitation, while $V$. pompona, $V$. odorata, $V$. insignis and $V$. inodora presented intermediate intervals. The amplitudes of extreme data for each species can be considered in locating areas where ex situ regional preservation strategies could be put in place, as well as in establishing areas for cultivation. The bioclimatic profile we found, allows for an indirect inference of each species' genetic condition, which could be used in genetic improvement programs; for instance, $V$. odorata grows at high altitudes and tolerates low temperatures, while $V$. inodora tolerates high temperatures, and $V$. odorata, V. pompona and $V$. planifolia tolerate low rain precipitation. Rev. Biol. Trop. 65 (3): 975-987. Epub 2017 September 01.
\end{abstract}

Key words: biodiversity, species, orchids, rain precipitation, temperature, altitude.

México es uno de los ocho centros de origen de varias especies comestibles cultivadas y silvestres, como el maíz, frijol, calabaza, chile, papaya, algodón y vainilla, las cuales han contribuido de manera sobresaliente a la alimentación y desarrollo de la humanidad (Ramírez et al., 2000; Caso \& Aliphat, 2006; Molina \& Córdova, 2006; CONABIO 
\& SEMARNAT, 2009). Conocer la distribución geográfica y las condiciones ambientales de las especies domesticadas y semidomesticadas es de vital importancia para el manejo integrado de especies con interés económico agrícola (Soto, 2009; Pliscoff \& Fuentes-Castillo, 2011; Álvarez, Osorio, \& Montoya, 2013). La prevalencia y domesticación de estas especies es un proceso que se ha dado en coevolución con el hombre, el cual se ha adaptado a distintas situaciones ambientales, regionales y culturales (Altieri \& Toledo, 2011; Cornejo \& Ibarra, 2011).

Vanilla es un género perteneciente a la familia Orchidaceae; se originó en América tropical hace 70 millones de años (Ramírez, Gravendeel, Singer, Marshall, \& Pierce, 2007; Bouétard et al., 2010); está compuesto por 110 especies distribuidas en las zonas tropicales de México y el mundo (Bory et al., 2008; Bouétard et al., 2010; Gigant, Bory, Grisoni, $\&$ Besse, 2011). Dentro de este género, Vanilla planifolia, Vanilla pompona y Vanilla tahitensis son las especies más importantes en el mundo, por ser productoras de vainillina natural (Bory et al., 2008; Álvarez et al., 2013; Azofeifa-Bolaños, Paniagua-Vásquez, \& García-García, 2014). La vainillina es considerada la sustancia aromatizante y saborisante más importante, con mayor valor económico en el mercado (Divakaran, Nirmal, \& Peter, 2006; Pérez-Silva et al., 2006; Pérez, Günata, Lepoutre, \& Odoux, 2011). A pesar de su importancia, muchas de las especies del género no se consideran domesticadas, las especies cultivadas son similares a sus parientes silvestres donde solo existe una selección empírica por parte de los productores.

En el cultivo de vainilla, su diversidad genética es limitada en las plantaciones comerciales, debido a la extensa propagación vegetativa del germoplasma (Besse et al., 2004; Bory et al., 2008; Lubinsky, Bory, Hernández, Kim, \& Gómez-Pompa, 2008). Una revisión de las especies del género Vanilla determinó la existencia de 15 especies de México y Centroamérica (Soto \& Dressler, 2010). Esto evidencia la diversidad biológica y cultural de la región como fuente de variación genética del género. A pesar de ello, las poblaciones naturales se encuentran en peligro de extinción, debido principalmente a la deforestación, colectas excesivas de material para el establecimiento de nuevas plantaciones y a inadecuados manejos del cultivo (Soto, 2006, 2009; Bory et al., 2008).

En México y Centroamérica, la vainilla representa un recurso fitogenético de suma importancia, ya que ahí existen el clima y suelo favorables para su cultivo, así como la cantidad y distribución adecuada de la precipitación a lo largo del año (ASERCA, 2002). En México, solamente $V$. planifolia se cultiva con fines comerciales, debido a que es la más demandada en la industria; su cultivo comercial se originó en la zona conocida como Totonacapan, que abarca parte de los estados de Veracruz y Puebla (Lubinsky et al., 2008; Reyes-López, Rodríguez, Kelso, Huerta, \& Ibáñez, 2008). Actualmente, en México existen plantaciones en Chiapas, Quintana Roo, San Luis Potosí, Hidalgo y Oaxaca (Soto, 2009; Hernández, Sánchez, Curti, \& Larios, 2010).

La descripción botánica de las especies de vainilla para México se menciona en Soto (2003) y en Soto y Dressler (2010); sin embargo, no se analiza la relación de la distribución de las especies con las variables bioclimáticas, que son de suma importancia para conocer dónde se encuentran las condiciones ambientales más adecuadas, para que una especie prospere, para análisis biogeográficos, ecológicos y de conservación (Anderson, Lew, \& Peterson, 2003; Beaumont, Hughes, \& Poulsen, 2005; Phillips, Anderson, \& Schapire, 2006).

Al considerar que la distribución de los organismos está determinada por el clima, es necesario su análisis para conocer porqué una especie crece en un determinado sitio y no en otro (Lindenmayer, Nix, McMahon, Hutchinson, \& Tanton, 1991). En este sentido Fischer, Lindenmayer, Nix, Stein, y Stein (2001) mencionan que cada especie tiene su propio perfil bioclimático, y el análisis de las variables que determinan dicho perfil, sirve para cuantificar las diferencias en los dominios climáticos que se presentan en las diferentes especies. 
En virtud de lo mencionado, el objetivo del presente trabajo fue conocer la diversidad del género Vanilla en México y su relación con algunas variables climáticas, con el fin de caracterizar parte del perfil bioclimático de las especies de vainilla. Estos conocimientos, contribuirán en la toma de decisiones en lo relacionado con su ecología, conservación y fitomejoramiento, al considerar los nuevos escenarios de cambio climático.

\section{MATERIALES Y MÉTODOS}

Para obtener datos de presencia de las diferentes especies de vainilla se realizaron salidas de campo, en el periodo comprendido entre 2008 y 2014, en sitios seleccionados a partir de información proveniente de herbarios e informantes clave, en los estados de Chiapas, Oaxaca, Puebla, Quintana Roo y Veracruz. En cada sitio, se recorrieron transectos de 5 $\mathrm{km}$, a través de senderos, caminos, parcelas y potreros. Se recolectaron entre tres y cinco ejemplares vivos de cada morfotipo de vainilla, localizado en cada sitio de muestreo; esta cifra varió, dependiendo del número de plantas encontradas. Se registraron datos generales de la localidad, donador, y con ayuda de un geoposicionador (GPS, marca Garmin), se tomaron las coordenadas en grados y altura sobre el nivel del mar. Los registros, se concentraron en hojas con datos pasaporte, recomendada por el Sistema Nacional de los Recursos Fitogenéticos de México (SINAREFI). Los ejemplares recolectados fueron cultivados en el Banco de Germoplasma de Vainilla de la Benemérita Universidad Autónoma de Puebla (BUAP), ubicado en Tenampulco, Puebla, bajo condiciones de igualdad en manejo agronómico, nutrición y sombra, según Hernández et al. (2010).

Para complementar la información sobre la diversidad de las especies de vainilla, durante el periodo de enero a mayo 2008 se realizó una consulta a los herbarios del Instituto Politécnico Nacional (IPN), Universidad Nacional Autónoma de México (MEXU) e Instituto Nacional de Ecología (XAL); además se consultó las bases de datos de la Red Mundial de Información sobre Biodiversidad de la CONABIO (REMIB) y del Global Biodiversity Information Facility (GBIF). Los datos de recolecta fueron introducidos a un sistema de información geográfica (ArcGis v.10.0) para la obtención de mapas de distribución de cada especie. De acuerdo con la información contenida en las fichas de las plantas de vainilla de los herbarios consultados, el período correspondiente de recolecta y herborización de las muestras incluyó las fechas desde 1932 hasta el 2004.

Para obtener los intervalos ambientales que determinan la diversidad de las especies del género Vanilla en México, se utilizaron las 19 variables bioclimáticas de World Clim (Hijmans, Cameron, Parra, Jones, \& Jarvis, 2005) y la altitud, a una resolución espacial de aproximadamente $1 \mathrm{~km}^{2}$ (Cuadro 1). Se realizó una extrapolación a cada punto de presencia, mediante análisis espacial de las diferentes variables ambientales, usando el SIG Idrisi (V.16.0). Se calcularon las medias, modas, desviaciones estándar y varianzas de las 19 variables de cada uno de los puntos registrados.

Se obtuvieron los intervalos de las condiciones ambientales extremas (mínimo, promedio y máximo) para cada una de las especies de vainilla por ubicación. Para determinar las variables de mayor importancia en la distribución de las especies, se realizó un análisis de componentes principales y en aquellas variables que resultaron significativas, se efectuaron pruebas de Kruskal-Wallis y Dunn, con la finalidad de delimitar el gradiente bajo el cual se encuentra cada especie. Para los análisis estadísticos se utilizó el software Statistica V.6.0 (Statsoft).

\section{RESULTADOS}

Se lograron registrar 288 puntos de recolecta, de los cuales, 188 fueron datos de campo y 100 de herbarios y base de datos internacionales. Con estos registros se obtuvieron mapas de distribución para $V$. planifolia, $V$. pompona, $V$. odorata, $V$. inodora, $V$. insignis, $V$. sprucei y V. cribbiana (Fig. 1). Chiapas fue el estado con mayor diversidad, con seis especies, seguido de Oaxaca, Puebla y Veracruz, con cinco especies 
CUADRO 1

Conjunto de 19 variables ambientales obtenidas de World Clim

TABLE 1

Set of 19 environmental variables from WorldClim

\begin{tabular}{cl} 
Abreviación & \\
BIO1 & Temperatura media anual \\
BIO2 & Intervalo de temperatura diurna media (Temp. máxima - Temp. mínima) \\
BIO3 & Isotermalidad (P2/P7) $*$ 100) \\
BIO4 & Estacionalidad de temperatura (desviación estándar *100) \\
BIO5 & Temperatura máxima del mes más caliente \\
BIO6 & Temperatura mínima del mes más frío \\
BIO7 & Intervalo de temperatura anual (Bio5-Bio6) \\
BIO8 & Temperatura media del trimestre más húmedo \\
BIO9 & Temperatura media del trimestre más seco \\
BIO10 & Temperatura media del trimestre más caliente \\
BIO11 & Temperatura media del trimestre más frío \\
BIO12 & Precipitación total anual \\
BIO13 & Precipitación del mes más húmedo \\
BIO14 & Precipitación del mes más seco \\
BIO15 & Estacionalidad de la precipitación (coeficiente de variación) \\
BIO16 & Precipitación del trimestre más húmedo \\
BIO17 & Precipitación del trimestre más seco \\
BIO18 & Precipitación del trimestre más caliente \\
BIO19 & Precipitación del trimestre más frío \\
\hline
\end{tabular}

cada uno; no obstante, los estados con mayor presencia de individuos fueron Quintana Roo y Chiapas con 83 y 66 registros, respectivamente, fueron nueve de los 32 estados de México donde se registró presencia de vainilla, Jalisco fue el estado donde se registró una sola especie, la cual fue $V$. pompona (Cuadro 2).
$V$. planifolia presentó un rango altitudinal promedio de 0 a 1650 m.s.n.m., mayor rango de adaptabilidad a la precipitación y temperatura, en comparación con las demás especies, la cual fue de 1021 a $4450 \mathrm{~mm}$ (BIO12), y de 16.7 a $27.8{ }^{\circ} \mathrm{C}(\mathrm{BIO} 1)$, respectivamente. $V$. odorata es la especie del género que presentó

\section{CUADRO 2}

Número de especies y número de registros del género Vanilla por estado

TABLE 2

Number of species and number of records of genus Vanilla by state

\begin{tabular}{llcc}
\multicolumn{1}{c}{ Estado } & \multicolumn{1}{c}{ Especies presentes } & Registros & Especies \\
Campeche & V. insignis, V. odorata. & 12 & 2 \\
Chiapas & V. cribbiana, V. inodora, V. odorata, V. planifolia, V. pompona, V. sprucei & 66 & 6 \\
Jalisco & V. pompona & 1 & 1 \\
Oaxaca & V. inodora, V. insignis, V. odorata, V. planifolia, V. pompona & 45 & 5 \\
Puebla & V. inodora, V. insignis, V. odorata, V. planifolia, V. pompona & 23 & 5 \\
Quintana Roo & V. insignis, V. odorata, V. planifolia, V. pompona & 83 & 4 \\
San Luis Potosí & V. odorata, V. planifolia & 2 & 2 \\
Tabasco & V. inodora, V. planifolia & 5 & 2 \\
Veracruz & V. inodora, V. insignis, V. odorata, V. planifolia, V. pompona & 51 & 5 \\
\hline
\end{tabular}



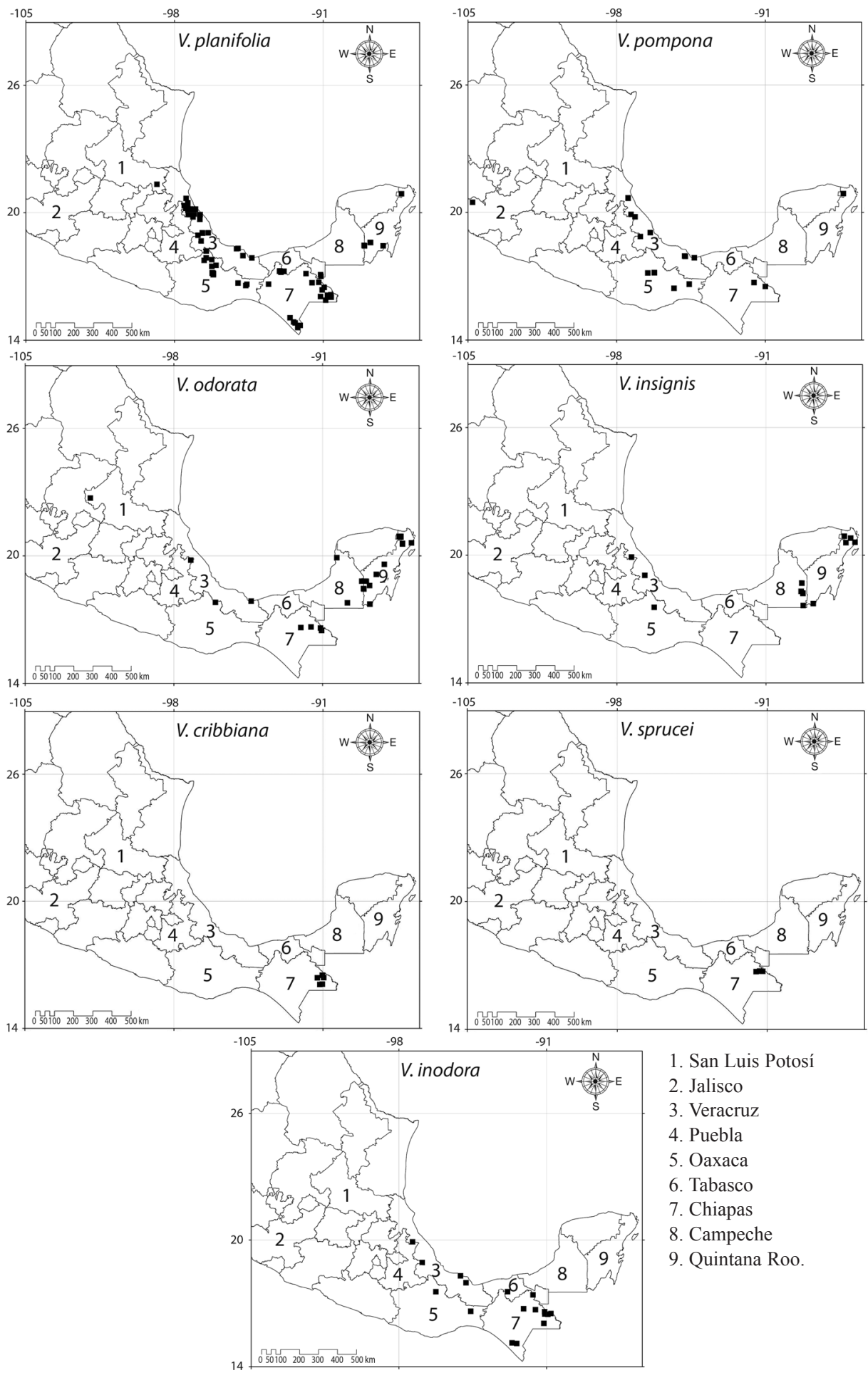

1. San Luis Potosí

2. Jalisco

3. Veracruz

4. Puebla

5. Oaxaca

6. Tabasco

7. Chiapas

8. Campeche

9. Quintana Roo.

Fig. 1. Distribución de la diversidad de especies del género Vanilla en México.

Fig. 1. Distribution of diversity of species from the genus Vanilla in México. 
los valores extremos más amplios en altitud, ya que se encontró entre los 0 y los 2065 m.s.n.m; así mismo, los valores más bajos en cuanto a temperaturas mínimas, fueron de 3.3 a $18.1^{\circ} \mathrm{C}$ (BIO6); sin embargo, su temperatura máxima del mes más cálido (BIO5) fue de $35.2{ }^{\circ} \mathrm{C}$.

$V$. pompona presentó un intervalo en altitud entre los 0 y los 1777 m.s.n.m, y ocupó el tercer lugar en altitud, en comparación con las demás especies; presentó el valor más bajo de temperatura promedio del mes más frío (BIO6), la cual fue de $13{ }^{\circ} \mathrm{C}$, y el rango de temperatura del mes más cálido, que osciló entre los 17.4 y $27.9^{\circ} \mathrm{C}(\mathrm{BIO} 10)$, toleró una precipitación media anual de 806 a $2477 \mathrm{~mm}$ (BIO12), que resultó baja, en comparación con las demás especies.

$V$. insignis presentó una altitud de 4 a 496 m.s.n.m (Cuadro 3), un amplio intervalo de precipitación media anual (BIO12), que es de
1068 a 3719 m.s.n.m, y valores extremos de temperatura media de 30.9 a $34.5{ }^{\circ} \mathrm{C}$ (BIO5). $V$. inodora es una especie que se adaptó a un rango de temperaturas máximas del mes más cálido de 26.5 a $35.5^{\circ} \mathrm{C}$. (BIO5); su tolerancia a la temperatura media del trimestre más seco fue de 16.2 a $27.7^{\circ} \mathrm{C}$ (BIO9); presentó una altitud intermedia, en comparación con las demás especies, que fue de 497 m.s.n.m.

V. cribbiana y V. sprucei son especies cuya presencia se limitó al estado de Chiapas, por lo que la amplitud de sus valores extremos tendió a ser más estrecha para la mayoría de las variables bioclimáticas (Cuadro 3).

Los resultados del análisis de componentes principales indicaron que el primer componente principal aportó el $38.2 \%$ de la varianza total, seguido de los componentes principales dos y tres, con el $26.7 \%$ y el $16.6 \%$ respectivamente, lo que resulta que la varianza acumulada

CUADRO 3

Condiciones ambientales extremas para el género Vanilla en México

TABLE 3

Extreme environmental conditions for genus Vanilla in Mexico

\begin{tabular}{|c|c|c|c|c|c|c|c|c|c|c|c|}
\hline Especie & Intervalo & Altitud & BIO1 & $\mathrm{BIO} 2$ & $\mathrm{BIO} 3$ & BIO4 & BIO5 & BIO6 & $\mathrm{BIO} 7$ & $\mathrm{BIO} 8$ & $\mathrm{BIO} 9$ \\
\hline \multirow[t]{3}{*}{ V. cribbiana } & Mínimo & 230 & 20 & 11 & 6.6 & 128.3 & 28.2 & 11.7 & 16.5 & 20.8 & 19.8 \\
\hline & Promedio & 514 & 24.2 & 11.4 & 6.8 & 155.2 & 32.6 & 15.9 & 16.7 & 25 & 24 \\
\hline & Máximo & 1277 & 25.6 & 12.1 & 7.3 & 167.7 & 34.2 & 17.1 & 17.1 & 26.4 & 25.5 \\
\hline \multirow[t]{3}{*}{ V. inodora } & Mínimo & 5 & 18.1 & 8.5 & 5.6 & 86.1 & 26.5 & 9.6 & 14.8 & 19.4 & 16.2 \\
\hline & Promedio & 497 & 23.8 & 11 & 6.5 & 174.1 & 32.3 & 15.5 & 16.9 & 24.8 & 23.5 \\
\hline & Máximo & 1424 & 26.6 & 14.2 & 7.7 & 307.4 & 35.5 & 18.1 & 19.4 & 27.7 & 27.7 \\
\hline \multirow[t]{3}{*}{ V. insignis } & Mínimo & 4 & 22.4 & 9.7 & 5.6 & 169.1 & 30.9 & 13.5 & 14.5 & 23.6 & 20 \\
\hline & Promedio & 139 & 24.9 & 10.9 & 6.4 & 206.3 & 33 & 16 & 17 & 26.3 & 23.7 \\
\hline & Máximo & 496 & 25.7 & 12.1 & 6.8 & 306.9 & 34.5 & 18.1 & 19.2 & 27.1 & 25 \\
\hline \multirow[t]{3}{*}{$V$. odorata } & Mínimo & 0 & 17.3 & 9.7 & 5.6 & 156.5 & 30.5 & 3.3 & 14.5 & 19.3 & 16.5 \\
\hline & Promedio & 130 & 25.1 & 11.2 & 6.6 & 192.2 & 33.3 & 16.4 & 16.9 & 26.6 & 24.4 \\
\hline & Máximo & 2065 & 26.8 & 18.6 & 7.1 & 291.5 & 35.2 & 18.1 & 27.2 & 28.2 & 26.5 \\
\hline \multirow[t]{3}{*}{ V. planifolia } & Mínimo & 0 & 16.7 & 8.5 & 5.1 & 51.1 & 25.9 & 7.5 & 13.4 & 17.1 & 15.7 \\
\hline & Promedio & 336 & 24 & 11.1 & 6.3 & 210.8 & 32.7 & 15.1 & 17.6 & 25.3 & 22.7 \\
\hline & Máximo & 1650 & 27.8 & 14.5 & 7.8 & 354.8 & 36.4 & 18.8 & 22.5 & 27.8 & 27.3 \\
\hline \multirow[t]{3}{*}{ V. pompona } & Mínimo & 0 & 15.7 & 9.2 & 5.1 & 137.1 & 25.5 & 5.8 & 16.1 & 16 & 15 \\
\hline & Promedio & 459 & 23 & 10.8 & 6 & 235 & 31.8 & 13.8 & 18 & 24.7 & 21.2 \\
\hline & Máximo & 1777 & 25.2 & 14.8 & 7 & 329.5 & 33.7 & 16.7 & 22.4 & 27.6 & 25 \\
\hline \multirow[t]{3}{*}{ V. sprucei } & Mínimo & 179 & 22.9 & 11.2 & 6.5 & 159.4 & 31.5 & 14.4 & 17.1 & 24.1 & 22.6 \\
\hline & Promedio & 503 & 24.5 & 11.6 & 6.7 & 172 & 33.2 & 16 & 17.2 & 25.5 & 24.2 \\
\hline & Máximo & 862 & 25.9 & 12 & 7 & 181.9 & 34.7 & 17.5 & 17.2 & 26.7 & 25.7 \\
\hline
\end{tabular}


CUADRO 3 (Continuación) / TABLE 3 (Continued)

\begin{tabular}{|c|c|c|c|c|c|c|c|c|c|c|c|}
\hline Especie & Rango & BIO10 & BIO11 & BIO12 & BIO13 & BIO14 & BIO15 & BIO16 & BIO17 & BIO18 & BIO19 \\
\hline \multirow[t]{3}{*}{ V. cribbiana } & Mínimo & 21.3 & 18 & 2097 & 370 & 34 & 58 & 1007 & 114 & 494 & 180 \\
\hline & Promedio & 25.9 & 21.9 & 2475 & 430 & 45 & 68 & 1143 & 168 & 563 & 262 \\
\hline & Máximo & 27.3 & 23.4 & 3010 & 460 & 79 & 74 & 1322 & 261 & 652 & 397 \\
\hline \multirow[t]{3}{*}{ V. inodora } & Mínimo & 20.1 & 15.5 & 1587 & 286 & 12 & 52 & 648 & 40 & 398 & 60 \\
\hline & Promedio & 25.7 & 21.3 & 2378 & 435 & 45 & 68 & 1113 & 158 & 536 & 252 \\
\hline & Máximo & 28.7 & 24.6 & 3719 & 688 & 75 & 89 & 1875 & 252 & 828 & 396 \\
\hline \multirow[t]{3}{*}{ V. insignis } & Mínimo & 24.7 & 19.1 & 1068 & 178 & 20 & 50 & 452 & 65 & 292 & 65 \\
\hline & Promedio & 27 & 21.9 & 1324 & 224 & 35 & 57 & 560 & 127 & 407 & 164 \\
\hline & Máximo & 27.4 & 23.2 & 3719 & 688 & 75 & 85 & 1875 & 252 & 828 & 335 \\
\hline \multirow[t]{3}{*}{ V. odorata } & Mínimo & 20.3 & 13.5 & 365 & 66 & 5 & 47 & 187 & 22 & 144 & 36 \\
\hline & Promedio & 27.1 & 22.4 & 1343 & 233 & 36 & 59 & 589 & 121 & 403 & 160 \\
\hline & Máximo & 29 & 23.8 & 2574 & 508 & 87 & 92 & 1325 & 265 & 645 & 300 \\
\hline \multirow[t]{3}{*}{ V. planifolia } & Mínimo & 18.5 & 14.6 & 1021 & 183 & 6 & 41 & 454 & 22 & 256 & 47 \\
\hline & Promedio & 26.2 & 21 & 2072 & 371 & 49 & 64 & 954 & 163 & 506 & 243 \\
\hline & Máximo & 29 & 26.7 & 4450 & 803 & 155 & 102 & 2061 & 491 & 946 & 878 \\
\hline \multirow[t]{3}{*}{$V \cdot$ pompona } & Mínimo & 17.4 & 13.8 & 806 & 184 & 5 & 45 & 511 & 17 & 177 & 18 \\
\hline & Promedio & 25.5 & 19.6 & 1650 & 308 & 38 & 70 & 783 & 127 & 452 & 180 \\
\hline & Máximo & 27.9 & 22.5 & 2477 & 473 & 78 & 112 & 1161 & 236 & 637 & 305 \\
\hline \multirow[t]{3}{*}{ V. sprucei } & Mínimo & 24.5 & 20.6 & 2218 & 406 & 46 & 62 & 996 & 175 & 516 & 258 \\
\hline & Promedio & 26.3 & 22 & 2419 & 432 & 52 & 63 & 1080 & 190 & 558 & 274 \\
\hline & Máximo & 27.9 & 23.2 & 2562 & 450 & 58 & 64 & 1142 & 205 & 591 & 285 \\
\hline
\end{tabular}

de los tres primeros componentes principales fuera el $81.5 \%$ de la variación total del conjunto de variables analizadas (Cuadro 4).

De acuerdo con los valores de los vectores propios (Cuadro 5) las variables que mayor aporte tuvieron en el primer componente principal fueron la altitud (-0.9), las variables BIO1, BIO5, BIO6, BIO8, BIO9 y BIO10 (0.9). En el segundo componente principal, fueron $\mathrm{BIO} 12$ y $\mathrm{BIO} 13$ (-0.9) y en el componente principal tres resultó $\mathrm{BIO} 3(-0.9)$ y $\mathrm{BIO} 4(0.9)$ (Cuadro 5). Al realizarles pruebas de Kruskal - Wallis y Dunn, se encontró que todas las variables consideradas tuvieron un comportamiento estadísticamente diferente $(\mathrm{P} \leq 0.05)$ para cada una de las especies de vainilla (Cuadro 6).

\section{DISCUSIÓN}

El hecho de haber encontrado registros del género Vanilla en nueve estados de la República Mexicana, nos indica que son lugares donde se presentan las condiciones favorables para el buen desarrollo de las plantas de vainilla cultivada y silvestre. De manera cultivada, se reportan siembras en los estados de Veracruz, Puebla, Oaxaca, Chiapas, Quintana Roo, San Luis Potosí

\section{CUADRO 4}

Valores propios, porcentaje absoluto y acumulado de la varianza para los tres componentes principales en el estudio de 19 variables bioclimáticas en vainilla

TABLE 4

Study results, absolute percentage, and accumulated percentage of variance for the three main components in the study with 19 genus Vanilla bioclimatic variables

\begin{tabular}{ccccc} 
Componente principal & Valor Propio & \% Total varianza & Valor Propio Acumulado & Varianza Acumulada\% \\
1 & 7.6 & 38.2 & 7.6 & 38.2 \\
2 & 5.3 & 26.7 & 13 & 64.9 \\
3 & 3.3 & 16.6 & 16.3 & 81.5 \\
\hline
\end{tabular}


CUADRO 5

Valores propios (Eigen vectores) de los primeros tres componentes principales para el estudio de 19 variables bioclimáticas en vainilla

TABLE 5

Study results (Eigen vectors) for the three main components in the study with 19 genus Vanilla bioclimatic variables.

\begin{tabular}{cccccccccccc} 
Comp. Principal & ALT & BIO 1 & BIO 2 & BIO 3 & BIO 4 & BIO 5 & BIO6 & BIO 7 & BIO 8 & BIO 9 \\
1 & -0.9 & 0.9 & -0.4 & 0.0 & 0.0 & 0.9 & 0.9 & -0.5 & 0.9 & 0.9 \\
2 & -0.0 & -0.0 & 0.1 & -0.1 & 0.2 & -0.1 & -0.2 & 0.2 & 0.0 & -0.3 \\
3 & 0.2 & 0.1 & 0.4 & 0.9 & -0.9 & -0.0 & 0.2 & -0.4 & -0.2 & 0.3 \\
Comp. Principal & BIO 10 & BIO 11 & BIO 12 & BIO 13 & BIO 14 & BIO 15 & BIO 16 & BIO 17 & BIO 18 & BIO 19 \\
1 & 0.9 & 0.9 & -0.1 & -0.2 & -0.1 & -0.4 & -0.2 & -0.0 & -0.2 & -0.0 \\
2 & -0.0 & -0.1 & -0.9 & -0.9 & -0.8 & 0.0 & -0.9 & -0.8 & -0.8 & -0.9 \\
3 & -0.2 & 0.4 & 0.1 & 0.2 & -0.5 & 0.6 & 0.2 & -0.5 & -0.1 & -0.2 \\
\hline
\end{tabular}

CUADRO 6

Prueba de medias para la altitud y variables bioclimáticas del género Vanilla

TABLE 6

Test of means for genus Vanilla altitude and bioclimatic variables

\begin{tabular}{|c|c|c|c|c|c|c|c|c|c|c|}
\hline Especie & Altitud & BIO 1 & $\mathrm{BIO} 4$ & BIO 5 & BIO 6 & BIO 8 & BIO 9 & BIO 10 & BIO 11 & BIO 12 \\
\hline V. planifolia * & $\mathrm{b}$ & a & a & a & a & a & a & a & $\mathrm{a}$ & $\mathrm{a}$ \\
\hline V. pompona $* *$ & $\mathrm{~b}$ & $\mathrm{~b}$ & $\mathrm{c}$ & $\mathrm{b}$ & $\mathrm{a}$ & $\mathrm{a}$ & b & a & $\mathrm{c}$ & $\mathrm{c}$ \\
\hline V. inodora** & $\mathrm{b}$ & b & b & a & b & $\mathrm{b}$ & $\mathrm{a}$ & $\mathrm{b}$ & $\mathrm{c}$ & $\mathrm{b}$ \\
\hline V. insignis** & d & $\mathrm{c}$ & $\mathrm{c}$ & d & $\mathrm{c}$ & $\mathrm{c}$ & $\mathrm{c}$ & d & d & $\mathrm{b}$ \\
\hline V. odorata** & $\mathrm{a}$ & $\mathrm{b}$ & $\mathrm{c}$ & $d$ & $\mathrm{a}$ & $\mathrm{b}$ & b & $\mathrm{b}$ & $\mathrm{b}$ & $\mathrm{b}$ \\
\hline V. cribbiana $* * *$ & $\mathrm{~b}$ & $\mathrm{c}$ & d & $\mathrm{c}$ & $\mathrm{c}$ & $\mathrm{c}$ & $\mathrm{c}$ & $\mathrm{c}$ & d & $\mathrm{c}$ \\
\hline V. sprucei*** & $\mathrm{c}$ & $\mathrm{c}$ & d & d & d & $\mathrm{c}$ & d & d & d & d \\
\hline
\end{tabular}

Medias con letras iguales no son estadísticamente diferentes (Kruskal-Wallis, 0.05) *Grupo estadístico a, ** Grupo estadístico bc, *** Grupo estadístico cd.

e Hidalgo (FAO, 2010; Hernández et al., 2010). Por otro lado, al considerar el aspecto comercial, la denominación de origen de $V$. planifolia, denominada vainilla de Papantla, se limita a 20 municipios de Veracruz y 19 del estado de Puebla, con localidades situadas geográficamente entre los 0 y 300 m.s.n.m., debido a que las características de sabor y aroma únicos de la vainilla de Papantla se encuentran determinados por factores naturales como altitud, clima y la presencia de vientos llamados nortes, mismos que consisten en frentes fríos que ingresan a la región, provenientes del noreste o del norte (IMPI, 2009). En este sentido, Pérez-Silva et al. (2011) reporta la presencia de 26 compuestos responsables del aroma a vainilla en extractos orgánicos de $V$. planifolia mexicana. Sabik, et al. (2016) reportan la presencia de 81 compuestos volátiles en frutos curados de $V$. planifolia de origen mexicano. Estos estudios revelan que la $V$. planifolia mexicana muestra alta calidad aromática, a nivel internacional, por lo que es preferida por los productores mexicanos para su cultivo en zonas donde presentan las condiciones climáticas favorables para su desarrollo, lo que explica su alta dispersión y adaptación en las diferentes zonas de cultivo en México.

El género Vanilla comprende alrededor de 107 especies distribuidas en climas tropicales del mundo (Soto \& Cribb, 2010). Para América 
tropical se reportan 52 especies (Gigant et al., 2011), para México y Centroamérica 15 diferentes especies (Soto \& Dressler, 2010) y para México Soto (2003) reporta las especies $V$. cribbiana Soto Arenas (Roed), V. hameri Soto Arenas (Roed), V. inodora Shiede, V. insignis Ames, $V$. odorata, $V$. perpleja Soto Arenas, $V$. phaeantha, V. planifolia G. Jackson, V. pompona Shiede, y una especie muy similar a $V$. planifolia denominada como $V$. Sp. M. Soto 8355; sin embargo, no se reportan datos de georreferenciación. Considerando únicamente los datos de herbarios y base de datos internacionales, se logró obtener registros de $V$. planifolia, $V$. pompona, V. odorata, V. inodora, V. insignis, $V$. sprucei y $V$. cribbiana, resultados que difieren con lo reportado por Soto (2003), debido a que en los herbarios de México y bases de datos internacionales, no se encontró registros de las especies $V$. ameri Soto Arenas (roed), $V$. perpleja Soto Arenas y $V$. phaeantha. Por otra parte, Soto (2003) no reporta a $V$. sprucei y $V$. cribbiana, registros que se encontraron para México en la base de datos de Global Biodiversity Information (GBIF).

Estos resultados permiten actualizar la información sobre el número de especies que puedan existir en México; no obstante, aún existe la posibilidad de encontrar más especies para México, debido a que no se ha recorrido todos los lugares de bosques y selvas donde posiblemente existan especies de vainilla creciendo de manera silvestre. De igual forma, se necesitan estudios de tipo botánico, caracterización morfológica y molecular para separar e identificar especies y clones dentro de especies, ya que en México hay muchas sinonimias locales como vainilla mansa, cimarrona, colibrí, tlatepusco, rayada, acamaya, entre otros, lo que ha generado que una misma especie sea referida con diferentes nombres. Por ejemplo, la vainilla colibrí es aplicada para nombrar a $V$. planifolia y también a $V$. odorata, $V$. pompona en algunos lugares de Oaxaca es llamada oreja de burro que es un cultivar de $V$. planifolia, lo que crea confusión taxonómica dentro del género Vanilla en México (Lubinsky et al., 2008).
$V$. planifolia, $V$. pompona y $V$. odorata fueron las especies con mayor número de presencias, y se encontraron en siete de los nueve estados de la República Mexicana donde se distribuye el género, esto debido principalmente a su semidomesticación en el manejo de su cultivo. Dos de estas tres especies presentan gran demanda en la industria (Reyes-López et al., 2008; Soto, 2009; Azofeifa-Bolaños et al., 2014), $V$. inodora y $V$. insignis se encontraron en cinco estados de México, mientras que $V$. sprucei y $V$. cribbiana solo se registraron en el estado de Chiapas, resultados que coinciden con lo reportado por Soto y Dressler (2010).

Lubinsky et al. (2008), mencionan que los cultivos de $V$. planifolia y $V$. pompona se originaron en México, probablemente en el norte de Veracruz. Vanilla planifolia es la especie que presenta mayor cantidad y calidad de compuestos aromáticos (ASERCA, 2002; Pak, Gropper, Dai, Havkin-Frenkel, \& Belanger, 2004; Hernández, 2011), por lo que es la que presenta mayor superficie sembrada (SAGARPA-SIAP, 2008), y ha sido objeto de domesticación mediante la selección empírica por parte de los productores, así como el diseminado como cultivo en los estados productores de México, es por eso que esta especie se encontró con mayor abundancia y con mayores rangos de amplitud en valores extremos.

$V$. pompona, es una especie que se encontró de manera silvestre y dentro de las plantaciones comerciales de $V$. planifolia, sus propiedades aromáticas son diferentes a $V$. planifolia y su mercado es más limitado (Gamboa-Gaitán, 2014); por otro lado, fue la segunda especie con mayor rango de amplitud en altitud (0 a 1777 m.s.n.m). Según Hernández et al. (2010) la altitud más favorable para el cultivo de $V$. planifolia es de 0 a 600 m.s.n.m., aunque Soto (2003), menciona que se han encontrado hasta los 1100 m.s.n.m., y en otros países como la India, se reportan hasta los 1500 m.s.n.m. (Anandaraj, Rema, Sasikumar, $\&$ Suseela-Bhai, 2005). En el presente trabajo se reporta que $V$. planifolia se encontró en promedio a una altura de 336 m.s.n.m., y un máximo de 1650 m.s.n.m; sin embargo, existieron 
otras especies como $V$. odorata, que se encontró a un máximo de 2065 m.s.n.m.; esta especie es la que se presentó a la mayor altitud en México.

$V$. planifolia, $V$. pompona y $V$. odorata presentaron valores mínimos en la variable BIO14, que corresponde a la precipitación del mes más seco, con 6.5 y $5 \mathrm{~mm}$, respectivamente, resultados que indican que estas especies pueden ser establecidas en localidades con baja precipitación; de estas tres especies es $V$. odorata la que requiere menor precipitación promedio anual en el mes más húmedo. Hernández et al. (2010) menciona que $V$. planifolia en México requiere una precipitación media anual de $1500 \mathrm{~mm}$, y con precipitaciones mayores a los $3000 \mathrm{~mm}$ anuales, las plantaciones presentan problemas de ataque de hongos. En este sentido Hágsater et al. (2005) y Tanaka y Kamemoto (1984), mencionan que la vainilla es una planta endémica de los bosques siempre verdes en regiones que reciben más de $2500 \mathrm{~mm}$ de precipitación anual.

Con relación a la temperatura, Hernández et al. (2010) menciona que $V$. planifolia prospera adecuadamente como cultivo con temperaturas promedio de 21 a $27^{\circ} \mathrm{C}$ con máximos de 32 ${ }^{\circ} \mathrm{C}$, las temperaturas menores de $7{ }^{\circ} \mathrm{C}$ provocan daños por quemaduras en la planta. En general, todas las especies se encontraron en localidades con promedios de temperatura anual entre $23 \mathrm{y}$ $25^{\circ} \mathrm{C}$; no obstante, en temperaturas máximas del mes más caliente, $V$. planifolia y $V$. inodora presentaron los valores máximos de tolerancia a temperaturas de 36.4 y $35.5{ }^{\circ} \mathrm{C}$, respectivamente. Las especies $V$. odorata, $V$. pompona y $V$. planifolia fueron las que presentaron los valores mínimos de temperatura del mes más frío con 3.3, 5.8 y $7.5{ }^{\circ} \mathrm{C}$, respectivamente. Estos valores de amplitud a los valores extremos en temperaturas y precipitación, nos permiten conocer la adaptabilidad y distribución, siendo las especies $V$. planifolia, $V$. odorata, y $V$. pompona las que presentaron mayores potencialidades en ser establecidas en lugares con poca precipitación en el mes más seco, y bajas temperaturas en el mes más frío.

Una forma para conservar la variabilidad genética del género Vanilla en México es mediante un banco de germoplasma, donde se resguardan in vivo cinco especies correspondientes a $V$. planifolia, $V$. pompona, $V$. inodora, $V$. insignis y $V$. odorata (Reyes-López, 2014, 2015); resta por encontrar las especies $V$. cribbiana y $V$. sprucei, que aun cuando se han visitado los lugares donde se reporta su existencia, no se ha logrado su rescate para su conservación; pero no se descarta su presencia, ya que aún no se han explorado totalmente los lugares donde posiblemente existan de manera silvestre.

Aun cuando $V$. planifolia, ha sido la especie más utilizada por los productores en sus siembras comerciales por sus cualidades aromáticas, esta se encuentra como sujeta a protección especial (PR), según la Norma Oficial Mexicana (NOM-059-SEMARNAT-2010), y es considerada por la FAO (1996) como especie amenazada; y la Convención sobre el Comercio Internacional de Especies Amenazadas de Flora y Fauna Silvestre (CITES-2015) considera a Orchidaceae spp. en el apéndice II, donde se contempla la vainilla. Por otro lado, Soto (2006), menciona que en México $V$. planifolia está en peligro de extinción y reporta tan solo 30 especímenes silvestres en la última década. Para la mayoría de las especies del género Vanilla en México, no se tiene registro de su estatus de conservación, por lo que es urgente la definición de estrategias de conservación in situ y ex situ, y considerar sus perfiles bioclimáticos, por ser una herramienta que ayuda a entender el comportamiento de desarrollo de una determinada especie (Lindenmayer et al., 1991; Anderson et al., 2003; Richardson \& Whittaker, 2010).

El perfil bioclimático encontrado en cada una de las especies de vainilla en México, permite conocer bajo qué situaciones se puede desarrollar cada especie, lo que es importante para su establecimiento como cultivo, debido a que los valores extremos de altitud, temperatura y precipitación son elementos que determinan su buen desarrollo vegetativo y calidad de los frutos. Por otro lado, estos conocimientos nos permiten inferir parte de la condición genética de cada especie, que puede 
ser utilizada en programas de mejoramiento genético. $V$. odorata es una especie con características genéticas para adaptarse a altitudes altas y tolerante a bajas temperaturas. $V$. inodora y $V$. planifolia presentaron valores de tolerancia a temperaturas altas que pueden ser utilizadas para la formación de híbridos con dichas características.

\section{AGRADECIMIENTOS}

Al Sistema Nacional de los Recursos Fitogenéticos de México (SINAREFI), por los apoyos económicos otorgados para la realización del presente trabajo. Al Consejo Nacional de Ciencia y Tecnología (CONACYT) por el apoyo económico otorgado con en número de beca 554375 .

\section{RESUMEN}

El género Vanilla comprende alrededor de 110 especies, distribuidas en las partes tropicales del mundo, y es el continente americano donde se encuentra la mayor cantidad de especies reportadas. En México, el cultivo de la vainilla está relacionado con diversas culturas como la totonaca, maya, chinanteca, mazateca, entre otras. En la actualidad, este cultivo presenta factores condicionantes tales como: técnicos, económicos, sociales, ecológicos y climáticos, que limitan su producción y la conservación de especies silvestres y cultivadas, por lo que es necesario, conocer su estado actual, en relación con su diversidad, así como algunos de los principales indicadores del perfil bioclimático de cada una de las especies, que ayuden en la toma de decisiones para su conservación y mejoramiento genético. Durante 2008, se realizaron consultas a herbarios de IPN, MEXU, XAL, base de datos de la Red Mundial de Información sobre la Biodiversidad de la CONABIO (REMIB), Global Biodiversity Information Facility (GBIF) $\mathrm{y}$ datos de accesiones vivas del banco de germoplasma de vainilla de la BUAP, formado del 2008 al 2014. Se realizaron mapas de distribución mediante un sistema de información geográfica. Se obtuvo el perfil bioclimático de cada especie considerando 19 variables de World Clim, y altitud a una resolución espacial de aproximadamente 1 $\mathrm{Km}^{2}$. Se calcularon las medias, desviaciones estándar y varianzas de las 19 variables en cada uno de los puntos registrados. Se obtuvieron los intervalos de las condiciones ambientales extremas (mínimo, promedio y máximo) para cada una de las especies de vainilla. Para determinar las variables de mayor importancia en la distribución de las especies se realizó un análisis de componentes principales, y a las variables que resultaron significativas se les realizó pruebas de Kruskal-Wallis y Dunn. Los resultados indicaron que en México se tienen registros de $V$. planifolia, $V$. pompona, $V$. insignis, $V$. inodora $V$. odorata, $V$. cribbiana y $V$. sprucei distribuidas en nueve estados. $V$. planifolia presentó intervalos amplios de temperatura y precipitación; $V$. pompona, $V$. odorata, $V$. insignis y $V$. inodora tuvieron intervalos intermedios. La amplitud de los datos extremos de cada especie puede considerarse para ubicar los sitios donde se puedan llevar a cabo estrategias regionales de conservación ex situ y el establecimiento de cultivos. El perfil bioclimático encontrado permite inferir de manera indirecta la condición genética de cada especie que podría ser utilizada en programas de mejoramiento genético como: la alta altitud y tolerancia a bajas temperaturas ( $V$. odorata), la tolerancia a altas temperaturas ( $V$. inodora) y tolerancia a baja precipitación ( $V$. odorata, $V$. pompona y V. planifolia).

Palabras clave: biodiversidad, especies, orquídeas, precipitación, temperatura, altitud.

\section{REFERENCIAS}

Altieri, M., \& Toledo, V. M. (2011). La revolución agroecológica de América Latina: Rescatar la naturaleza, asegurar la soberanía alimentaria y empoderar al campesino. El Otro Derecho, 42, 163-202.

Álvarez, L. C. L., Osorio, N. W. V., \& Montoya, M. M. (2013). Identificación molecular de microorganismos asociados a la rizosfera de plantas de vainilla en Colombia. Acta Biológica Colombiana, 18, 293-305.

Anandaraj, M., Rema, J., Sasikumar, B., \& Suseela-Bhai, R. (2005). Vanilla (Extensión pamphlet). E. Odoux \& M. Grisoni. Institute of Spices Research. Calicut, Kerala, India: Taylor and Francis Group LLC.

Anderson, R. P., Lew, D., \& Peterson, A. T. (2003). Evaluating predictive models of species distributions: criteria for selecting optimal models. Ecological Modelling, 162, 211-232.

ASERCA. (2002). La Vainilla una tradición con alto potencial. Revista Claridades, 101.

Azofeifa-Bolaños, J. B., Paniagua-Vásquez, A., \& GarcíaGarcía, J. A. (2014). Importancia y desafíos de la conservación de Vanilla spp. (Orquidaceae) en Costa Rica. Agronomía Mesoamericana, 25, 189-202.

Beaumont, L. J., Hughes, L., \& Poulsen, M. (2005). Predicting species distributions: use of climatic parameters in BIOCLIM and its impact on predictions of species current and future distributions. Ecological Modelling, 186, 250-269.

Besse, P., Da Silva, D., Bory, S., Grisoni, M., Le Bellec, F., \& Duval, M. F. (2004). RAPD genetic diversity in cultivated Vanilla: Vanilla planifolia, and relationships 
with $V$. tahitensis and $V$. pompona. Plant Science, 167, 379-385.

Bory, S., Lubinsky, P., Risterucci, A. M., Noyer, J. L., Grisoni, M., Duval, M. F., \& Besse, P. (2008). Patterns of introduction and diversification of Vanilla planifolia (Orchidaceae) in Reunion Island (Indian Ocean). American Journal of Botany, 95, 805-815.

Bouétard, A., Lefeuvre, P., Gigant, R., Bory, S., Pignal, M., Besse, P., \& Grisoni, M. (2010). Evidence of transoceanic dispersion of the genus Vanilla based on plastid DNA phylogenetic analysis. Molecular Phylogenetics and Evolution, 55, 621-630.

Caso, B. L., \& Aliphat, F. M. (2006). Cacao, vanilla and annatto: three production and exchange systems in the Southern Maya lowlands, XVI-XVII centuries. Journal of Latin American Geography, 5, 29-52.

CONABIO \& SEMARNAT. (2009). Cuarto Informe Nacional de México al Convenio Sobre Diversidad Biológica (CDB). México D.F.: Comisión Nacional Para el Conocimiento y uso de la Biodiversidad.

Cornejo, G., \& Ibarra, G. (2011). Diversidad y distribución del género Salvia (Lamiaceae) en Michoacán, México. Revista Mexicana de Biodiversidad, $82,1279-1296$.

Divakaran, M., Nirmal, K., \& Peter, K. V. (2006). Conservation of Vanilla species, in vitro. Scientia Horticulturae, 110, 175-180.

FAO (Food and Agriculture Organization of the United Nations). (1996). Informe sobre el estado de los recursos fitogenéticos en el mundo. Leipzig. Alemania: Organización de las Naciones Unidas para la Agricultura y la Alimentación.

FAO (Food and Agriculture Organization of the United Nations). (2010). Consulta de bases de datos de producción mundial y comercio internacional de Vainilla. Recuperado de http://apps.fao.org/faostat

Fischer, J., Lindenmayer, D. B., Nix, H. A., Stein, J. L., \& Stein, J. A. (2001). Climate and animal distribution: a climatic analysis of the Australian marsupial Trichosurus caninus. Journal of Biogeography, 28, 293-304

Gamboa-Gaitán, M. A. (2014). Vainillas colombianas y su microbiota II. Diversidad, cultivo y microorganismos endófitos. Universitas Scientiarum, 19, 287-300.

Gigant, R., Bory, S., Grisoni, M., \& Besse, P. (2011). Biodiversity and evolution in the Vanilla genus. En O. Grillo \& G. Venora, (Eds.). The dynamical processes of biodiversity - case studies of evolution and spatial distribution (pp.1-26). Francia: InTech.

Hágsater, E., Soto, A. M. A., Salazar, G. A., Jiménez, R., López, M. A., \& Dressler, R. L. (2005). Las Orquídeas de México. México D.F.: Instituto Chinoín.
Hernández, J. (2011). Paquete tecnológico vainilla (Vanilla planifolia Jackson). Establecimiento y mantenimiento (Programa Estratégico para el desarrollo Rural Sustentable de la Región Sur-Sureste: Trópico Húmedo). Tlapacoyan, Veracruz: SAGARPA-INIFAP.

Hernández, J., Sánchez, M. S., Curti, D. E., \& Larios, R. M. (2010). La producción de vainilla en México (Libro técnico Núm. 25). INIFAP. Centro de Investigación Regional Golfo Centro.

Hijmans, R. J., Cameron, S. E., Parra, J. L., Jones, P. G., \& Jarvis. A. (2005). Very high resolution interpolated climate surfaces for global land areas. International Journal of Climatology, 25,1965-1978.

IMPI (Instituto Mexicano de la Propiedad Industrial). (2009, 5 marzo). Declaratoria general de protección de la denominación de origen vainilla de Papantla. Diario oficial de la federación, México, pp 107-109.

Lindenmayer, D. B., Nix, H. A., McMahon, J. P., Hutchinson, M. F., \& Tanton, M. T. (1991). The conservation of Lleadbeater's possum, Gymnobelideus leadbeateri (McCoy): A case study of the use of bioclimatic modelling. Journal of Biogeography, 18, 371-383.

Lubinsky, P., Bory, S., Hernández, J. H., Kim, S. Ch., \& Gómez-Pompa, A. (2008). Origins and dispersal of cultivated Vanilla (Vanilla planifolia Jacks. [Orchidaceae]). Economic Botany, 62, 127-138.

Molina, M. J. C., \& Córdova, T. L. (2006). Recursos fitogenéticos en México. Para la alimentación y la agricultura. Informe nacional 2006. Chapingo, Edo. de México.

Pak, F. E., Gropper, S., Dai, W. D., Havkin-Frenkel, D., \& Belanger, F. C. (2004). Characterization of a multifunctional methyltransferase from the orchid Vanilla planifolia. Plant Cell Reports, 22, 959-966.

Pérez-Silva, A., Günata, Z., Lepoutre, J. P., \& Odoux, E. (2011). New insight on the genesis and fate of odoractive compounds in vanilla beans (Vanilla planifolia G. Jackson) during traditional curing. Food Research International, 44, 2930-2937.

Pérez-Silva, A., Odoux, E., Brat, P., Ribeyre, F., RodriguezJimenes, G., Robles-Olvera, V., García-Alvarado, A., \& Günata, Z. (2006). GC-MS and GC-olfactometry analysis of aroma compounds in a representative organic aroma extract from cured vanilla (Vanilla planifolia G. Jackson) beans. Food Chemistry, 99, 728-735.

Phillips, S. J., Anderson, R. P., \& Schapire, R. E. (2006). Maximum entropy modeling of species geographic distributions. Ecological Modelling, 190, 231-259.

Pliscoff, P., \& Fuentes-Castillo, T. (2011). Modelación de la distribución de especies y ecosistemas en el tiempo y en el espacio: una revisión de las nuevas 
herramientas y enfoques disponibles. Revista de Geografia Norte Grande, 48, 61-79.

Ramírez, P., Ortega, R., López, A., Castillo, F., Livera, M., Rincón, F., \& Zavala, F. (2000). Sistema nacional de los recursos fitogenéticos. Chapingo, México.

Ramírez, R. S., Gravendeel, B., Singer, R. B., Marshall. C. R., \& Pierce, N. E. (2007). Dating the origin of the Orchidaceae from a fossil orchid with its pollinator. Nature, 448, 1042-1045.

Reyes-López, D., Flores, A., Huerta, M., Kelso, H. A., Avendaño, C. H., Ortiz, R., \& López, J. F. (2014). Variación morfométrica de fruto y semilla en cuatro especies del género Vanilla. Ecosistemas y Recursos Agropecuarios, 1, 205-218.

Reyes-López, D., Rodríguez, B., Kelso, H. A., Huerta, M., \& Ibáñez, A. (2008). Beneficiado Tradicional de Vainilla. Puebla, México: Benemérita Universidad Autónoma de Puebla.

Reyes-López, D., Quiroz-Valentín, J., Kelso-Bucio, H. A., Huerta-Lara, M., Avendaño-Arrazate, C. H., \& Lobato-Ortiz, R. (2015). Caracterización estomática de cinco especies del género Vanilla. Agronomía Mesoamericana, 26, 237-246.

Richardson, D. M., \& Whittaker, R. J. (2010). Conservation biogeography-foundations, concepts and challenges. Diversity and Distributions, 16, 313-320.

Sabik, H., Pérez-Silva, A., Bélanger, D., Vivar-Vera, M. de los Á., Nicolás-García, M., \& Reyes- López, D. (2016). Identification of volatile compounds in cured Mexican vanilla (Vanilla planifolia G. Jackson) beans using headspace solid-phase microextraction with gas chromatography-mass spectrometry. Fruits, 71(6), 1-11.
SAGARPA-SIAP. (2008). Consulta de base de datos de producción, superficie, rendimiento y precio de productos agropecuarios. Sistema agropecuaria de consulta (SIACON), 1980-2009. Versión Electrónica. México.

Secretaría de Medio Ambiente y Recursos Naturales (SEMARNAT). (2010, 30 de diciembre). NOM059-SEMARNAT-2010. Protección ambiental- especies nativas de México de flora y fauna silvestre - categoría de riesgo y especificaciones para su inclusión, exclusión o cambio - lista de especies en riesgo. Diario Oficial de la Federación, México, p. 77.

Soto, M. A. (2003). Vanilla. En M. A. Pridgeon, P. J. Cribb, M. W. Chase, \& F. M. Rasmussen (Eds.), Orchids of México, parts 2 and 3. Icones Orchidacearum fasc. 5-6 (pp. 321-334). México: Herbario AMO.

Soto, M. A. (2006). La vainilla: retos y perspectivas de su cultivo. Biodiversitas, 66, 1-9.

Soto, M. A. (2009). Recopilación y análisis de la información existente sobre las especies mexicanas del género Vanilla. Reporte. México, D. F.: Herbario de la Asociación Mexicana de Orquideología, A. C., Instituto Chinoín, A. C.

Soto, M. A., \& Cribb, P. (2010). A new infrageneric classification and synopsis of the genus Vanilla Plum. ex Mill. (Orchidaceae: Vanillinae). Lankesterian International Journal on Orchidology, 9, 355-398.

Soto, M. A., \& Dressler, R. L. (2010). A revision of the Mexican and Central American species of Vanilla plumier ex Miller with a characterization of their ITS region of the nuclear ribosomal DNA. Lankesteriana International Journal on Orchidology, 9, 285-354.

Tanaka, R., \& Kamemoto, H. (1984). Chromosomes in orchids: counting and numbers. En J. Arditti (Ed.), Orchid Biology: Reviews and Perspectives Vol. III (p. 324-410) Ithaca, USA.: Cornell University Press. 